\title{
An innovative tool for the evaluation and comparison of different fuels and technologies onboard ships
}

\author{
Diego Rattazzi ${ }^{1 *}$, Massimo Rivarolo ${ }^{1}$, and Aristide F. Massardo ${ }^{1}$ \\ ${ }^{1}$ Thermochemical Power Group, DIME - University of Genoa, Via Montallegro 1, 16145 Genova, Italy
}

\begin{abstract}
The paper focuses on the analysis of innovative energy systems onboard ships with the aim to evaluate, in a preliminary stage, which can be the most promising solution depending on the considered application. For this purpose, the dedicated tool HELM developed by the authors' research group is employed. The tool uses maps reporting the main indicators (weight, volume, costs and emissions) for each component in relation to the installed power and the operational hours required (given by the user as an input), then it compares the results providing the best solution depending on the considered application. The maps have been built from a database developed throughout a wide analysis of the available market solutions in terms of energy generation devices (i.e. fuel cells, internal combustion engines), fuels (hydrogen, natural gas, diesel, methanol) and related storage technologies. The main strong point of HELM resides in its flexibility: it can be used for different typologies and sizes of ships (e.g. ferry boat, cruises, yachts); moreover, the database can be easily updated with more technologies. In this work, the focus is particularly on hydrogen application with PEM Fuel Cells and the use of innovative fuels (methanol, ammonia) in Internal Combustion Engines. Analysing different applications, it will be highlighted how the specific characteristics and priorities of the application affect the results of the best solutions. Furthermore, considering the regulation roadmap for the next years in the maritime context, promising technologies are highlighted also for future scenarios.
\end{abstract}

\section{Introduction}

Nowadays, ship transportation represents the most efficient solution for the distribution of large amounts of goods for long distances. In 2018, shipping has confirmed its primary role, with 11,000 Mtons delivered, that corresponds to $90 \%$ of world transportation in weight terms [[1]]. About $99 \%$ of the world's fleet is powered by Internal Combustion Engines (ICEs) fuelled by fossil derivative fuels [1], in particular Heavy Fuel Oil (HFO) and Marine Gas Oil (MGO). Since the intensive use of oil-derived fuels, maritime transportation is nowadays responsible for about 870 Mtons per year, equivalent to $2.8 \%$ of the world's anthropogenic $\mathrm{CO}_{2}$ emissions [2]. Furthermore, the use of these fuels has an impact also in terms of pollutants such as SOx, NOx and particulate matter.

The International Maritime Organization (IMO) performed many studies and adopted different regulations in the last years in order to reduce the impact of maritime transports, defining low Emission Control Areas (ECAs), for both NOx (NECAs) and SOx (SECAs) emission control. In 2018, the IMO set the new long-term strategy for 2050, aimed at reducing by $50 \%$ $\mathrm{CO}_{2}$ emissions, compared to levels of 2008 [3]. In order to reach this ambitious target, the introduction of innovative and sustainable technologies for ship propulsion, combined with the substitution of HFO with more low environmental impact fuels is one of the hey points [4]. In this context, the introduction of PEM Fuel Cells, fuelled by pure hydrogen, for maritime applications are one of the most promising solutions, as they are a zero-emission system [4][6]. On the other hand, the impact that volumes and weights have in maritime applications cannot be neglected [7]: compared to other fuels, $\mathrm{H}_{2}$ presents a low energy density, resulting in practical drawbacks related to its storage on board, if compared with the currently employed liquid fuels, such as MGO and HFO [8]. Fig. 1 reports a comparison for different fuels. The graph compares the solutions considering both the gravimetric and volumetric densities, taking into account the storage systems as well. Although the superiority in terms of volume of diesel fuels is evident, there are some potential candidates, such as ammonia and methanol, which would allow for higher volumetric energy density

* Corresponding author: diego.rattazzi@edu.unige.it 
compared to hydrogen and natural gas. On the other hand, fuels that require cryogenic systems for liquefaction (i.e. $\mathrm{LNG}$ and $\mathrm{LH}_{2}$ ) present a drawback in terms of volumetric density.

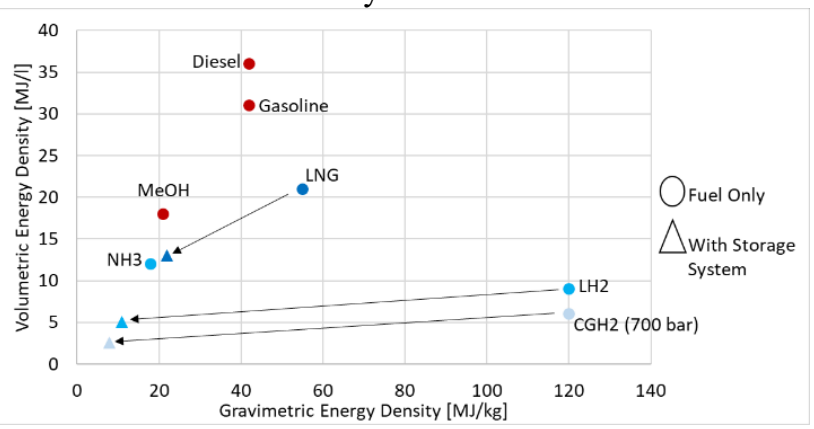

Fig. 1 Energy density for different fuels

However, the energy density in volume terms is not the only parameter that has to be evaluated. In general, considering propulsion solutions for maritime transportation and the related fuel storage systems, four main aspects have to be considered: (i) volumes; (ii) weights; (iii) investment and operational costs; (iv) $\mathrm{CO}_{2}$ and pollutant emissions, considering the recent IMO regulations. In order to compare the several possible solutions, the development of affordable tools is a key point, in particular in a preliminary design phase. Since the maritime sector covers a wide range of ships with different purposes and sizes, (i.e. yachts, cruise ferries, bulk carrier, etc.) the development of flexible tools to individuate the most suitable solutions for different scenarios and applications play a primary importance role. Recently, the author's research group [9][10] has developed a software tool named HELM (Helper for Energy Layouts in Maritime applications) for this purpose, applying it in some recent works to compare H2 PEM fuel cells with currently employed solutions, in particular internal combustion engines fuelled by HFO and LNG [8]. In the present research work, innovative solutions, based on ammonia and methanol fuels, which are attracting research in these years [2][5], are implemented in the tool and investigated considering different applicative cases.

\subsection{Methanol}

Methanol $\left(\mathrm{CH}_{3} \mathrm{OH}\right)$ is liquid at ambient pressure and temperature, making it much easier to manage and store onboard compared to gaseous fuels (i.e. $\mathrm{H}_{2}, \mathrm{CH}_{4}$ ), which require pressurization and/or refrigeration techniques in order to store it in liquid form. Methanol has the highest hydrogen to carbon $(\mathrm{H} / \mathrm{C})$ ratio among liquid fuels, thus allowing for a significant reduction in terms of $\mathrm{CO}_{2}$; this aspect has recently attracted the attention of many companies in the maritime sector. Furthermore, methanol does not contain sulphur, thus it does not present SOx emissions. Methanol has a Lower Heating Value (LHV) and an energy density lower than traditional marine fuel oils (HFO and MGO): with the same energy required, the use of methanol needs a storage volume 2.5 times higher than the one for traditional marine fuels. This aspect is partially compensated by methanol high octane number, which allows for more complete combustion and higher efficiency. The retrofitting of infrastructure used for conventional marine fuels to methanol is possible with minor modifications. Recently, the Stena Germanica RoPax ferry was retrofitted to run its engines (24 MW) with a mix of $95 \%$ methanol and $5 \%$ MGO. The ship represents the world's first methanol powered ferry [11].

\subsection{Ammonia}

Ammonia $\left(\mathrm{NH}_{3}\right)$ is a chemical compound based on nitrogen and hydrogen, naturally present in the gaseous state at ambient pressure and temperature. It can be easily stored as a liquid in pressurized tanks at ambient temperature and modest pressure (10 bar) or at ambient pressure by refrigeration $\left(-33^{\circ} \mathrm{C}\right)$. The second solution is the most employed at an industrial scale, as it presents lower initial investments.

Liquefied ammonia is an easy solution for transportation and storage, as it is not flammable and it does not show a tendency to ignition.

Ammonia has an LHV comparable to methanol and its volumetric energy density is lower than the one of traditional marine fuels: for the same amount of energy stored, $\mathrm{NH}_{3}$ storage requires a space 2.5 times larger than HFO and MGO, which represent the state of the art. $\mathrm{NH}_{3}$ utilization is an interesting solution from the environmental standpoint, as it does not contain carbon, thus it presents zero $\mathrm{CO}_{2}$ emissions in its combustion. Moreover, it does not present SOx, particulate matter and $\mathrm{CO}$ pollutant emissions. Ammonia is identified as one of the most interesting alternative fuels in a longterm scenario (2050) by IMO, as it would allow for reducing GHG emissions, as it is carbon-free. Although it has remarkable potential, its combustion in internal combustion engines needs an energy input for the ignition and heavy modifications to the engine. Furthermore, its combustion generates emissions in terms of NOx, therefore a system to manage the NOx is mandatory in order to respect the limits set by IMO.

Table 1 reports the main features for methanol and ammonia.

Table 1 Methanol and ammonia features

\begin{tabular}{|c|c|c|}
\hline Chemical composition & $\mathrm{CH}_{3} \mathrm{OH}$ & $\mathrm{NH}_{3}$ \\
\hline Boiling temperature $\left[{ }^{\circ} \mathrm{C}\right]$ at $1 \mathrm{bar}$ & 65 & -33 \\
\hline $\mathrm{LHV}[\mathrm{MJ} / \mathrm{kg}]$ & 20 & 22.5 \\
\hline Energy density [MJ/l] & 15.7 & 15.7 \\
\hline $\begin{array}{l}\text { Volume required factor } \\
\text { (compared with HFO) }\end{array}$ & 2.55 & 2.55 \\
\hline $\mathrm{kg} \mathrm{CO}_{2} / \mathrm{kWh}_{\mathrm{el}}$ & 0.25 & - \\
\hline $\begin{array}{l}\mathrm{kg} \mathrm{CO}_{2} / \mathrm{kWh}_{\text {ell }} \text { reduction } \\
\text { (compared with } \mathrm{HFO} \text { ) }\end{array}$ & $-11 \%$ & $-100 \%$ \\
\hline
\end{tabular}




\section{HELM tool description}

HELM is based on a rich database, which collects information on power units, fuels and storage systems, obtained through extensive market research and discussion with companies active in the maritime sector. The data put together in the database have been suitably processed to create maps that allow the software to assign a weight, volume and cost to the size of the system, entered as input by the user. Fig. 2 reports an example of the map present inside the tool (Fuel oil storage).

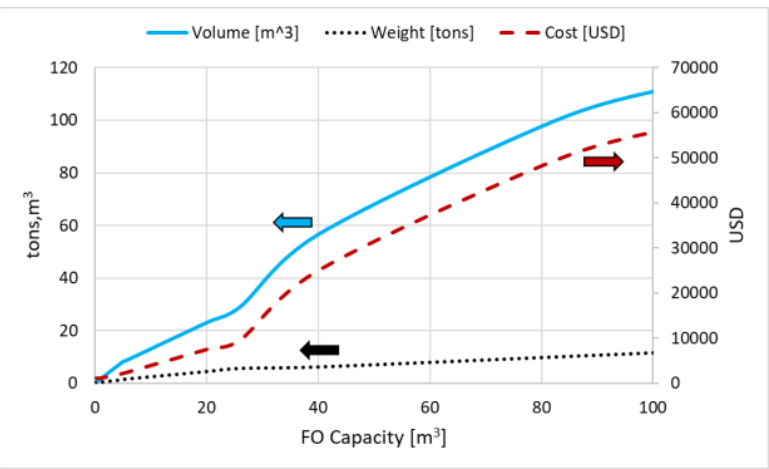

Fig. 2 Fuel oil storage maps

When entering the abscissa with the required fuel capacity, the mass and overall dimensions of the storage units are obtained on the left ordinates' axis, while the right ordinates axis provides the corresponding cost. It is noted that the curves have different gradients as fuel capacity varies: this is due to the use of different specific functions extrapolated from market analysis data.

Fig. 3 illustrates the logical scheme used by the software for processing the calculations.

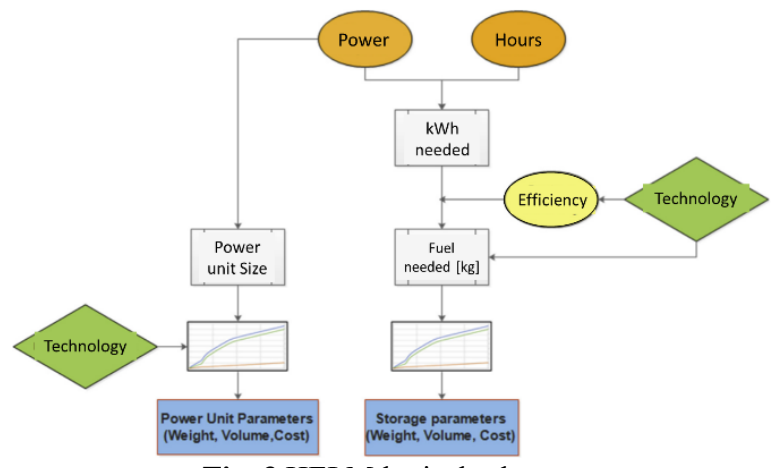

Fig. 3 HELM logical scheme

The main input data are the hours of non-stop navigation and the power required; to make a precautionary sizing, the maximum power required by the operational profile of the ship is entered, even if the power delivered for most of the time will be lower. The product of required power and operating hours returns the energy consumed by the vessel during navigation; dividing this value by the reference efficiency of the technical solution considered, the quantity of fuel required is obtained, with which the maps can be accessed to obtain the costs, weights and overall dimensions of the storage units. The general parameters of the power unit are obtained with an immediate procedure, as the size and costs can be obtained by entering the maps directly with the required power. This procedure is repeated for each technical solution implemented in the software.

In order to identify the best technical solution, the software assigns to each technology a score, which takes into account the performance in terms of weight, volume, cost and emissions: for each of these parameters (e.g. the total weight of the system), a score from 1 to 10 is assigned. The maximum score is given to the technical solution with the highest performance value (in the case of weight, therefore, the lightest system is assigned 10 points).

The points of the other technologies are assigned by comparison with the technology that has received the highest evaluation: the parameter $\mathrm{X}_{\mathrm{i}}=\mathrm{V}_{\mathrm{i}} / \mathrm{V}_{\text {best }}$ is calculated, where there is the value reported by the $\mathrm{i}$-th technology, while $\mathrm{V}_{\text {best }}$ is the value reported by the optimal solution; the score of the i-th technology will be assigned according to the range in which the $\mathrm{X}_{\mathrm{i}}$ parameter of the $\mathrm{i}$-th technology is placed. As an example, Fig. 4 shows the range of $X_{i}$ used to assign the points related to the evaluation of system performance in terms of weight.

\begin{tabular}{lccc}
\hline Value & Score & Value & Score \\
\hline $1<\mathrm{X} \leq 1.1$ & 10 & $3<\mathrm{X} \leq 4$ & 5 \\
$1.1<\mathrm{X} \leq 1.3$ & 9 & $4<\mathrm{X} \leq 5$ & 4 \\
$1.3<\mathrm{X} \leq 1.6$ & 8 & $5<\mathrm{X} \leq 6$ & 3 \\
$1.6<\mathrm{X} \leq 2$ & 7 & $6<\mathrm{X} \leq 8$ & 2 \\
$2<\mathrm{X} \leq 3$ & 6 & $\mathrm{X}>8$ & 1 \\
\hline
\end{tabular}

Fig. 4 Ranges of $X$ parameters for evaluation of the performance in terms of weight

Depending on the scenario considered for the analysed vessel, it is possible to set a specific relevance to each one of the performance parameters (weight, volume, costs and emissions). This allows characterizing the result of the best solution specifically in the context assigned. For each performance parameters, it is possible to give relevance between 1 and 5. The highest the value of the relevance for a performance parameter, the larger the priority given to this parameter during the evaluation process.

Generally, during the preliminary design phases of a vessel, the choice of the best applicable technical solution to produce energy onboard is mainly influenced by the weight, the volume occupied, the cost and the related pollutant emissions. The priority given to each of these performance parameters depends on the context in which the ship is to operate: if constant navigation in ECA zones is required, the environmental parameter will have a higher weight than the others; if, on the other hand, the ship is designed for the transport of goods, greater priority will be given to the compactness of the installed systems.

Furthermore, by selecting the ship size on the tool, it is also possible to consider if the volume and weights of the technology are feasible with the available size of the considered vessel. Among the strengths of HELM, it is worth mentioning its ease of use and the possibility to update the data to the technical innovations progressively introduced in the market.

\section{Case studies description}


Within the present study, HELM is used to evaluate the best solutions applicable for powering the hotel loads of a cruise ship for the duration of the stopover in port. Served by the ship's low-voltage electrical system, hotel loads include passenger and crew services and onboard auxiliary services. To define the data required as input by HELM, reference was made to the Costa Diadema, in navigation since October 2014 [12][13]. The technical specifications of the vessel are given in Table 2.

Table 2 Costa Diadema tech specifications [12][13]

\begin{tabular}{|l|l|}
\hline Gross Tonnage & 132500 \\
\hline Length over all [m] & 306 \\
\hline Passengers & 4947 \\
\hline $\begin{array}{l}\text { Generation total output } \\
{[\text { kW] }}\end{array}$ & 67200 \\
\hline Engines & $\begin{array}{l}4 x \text { Wartsila 12V46C } \\
2 x \text { Wartsila 8L46C }\end{array}$ \\
\hline
\end{tabular}

The ship is divided into 7 Main Vertical Zones (MVZ), i.e. vertical sectors designed to confine damage caused by accidents (e.g. flooding, fires) to the affected area only, safeguarding areas not affected. In order to size the generation system that takes charge of the hotel services demand, it is necessary to know the power loads: the power required by the Diadema during the day reaches 8.5 MW, while the annual average is $7.5 \mathrm{MW}$. As a precautionary measure, an installed capacity of $9 \mathrm{MW}$ is considered, which guarantees the operation of the services even in the heaviest conditions, i.e. in the warmer season, when the power absorbed by the air conditioning systems is maximum. The average length of stopover in ports is 12 hours, thus the installed systems must be able to supply the hotel load for 15 hours to be conservative. However, in order to guarantee an autonomy for two stopovers, the sizing of the storage systems is carried out considering 30 hours of autonomy. For what concerns the relevance of the performance parameters, considering the scenario of this application, the following values are considered:

- Volume (3): for a cruise ship, it is preferable to use compact systems in order to maximize the space available for rooms and hotel services

- Weight (1): since it is not a critical point for a vessel, its relevance is set to the lowest value.

- Cost (3): in a commercial application, the limitations of cost is important in order to make feasible the investment and the management of a cruise ship.

To assess the performance of the different technologies, two distinct scenarios are considered:
Case 1: low priority is given to the reduction of pollutant emissions, as typically happens in today's regulatory context (Emissions relevance: 2).

Case 2: the environmental impact of the solutions applied is more strictly controlled (Emissions relevance: 5 ).

Case 3: In this last case, each MVZ is equipped with a dedicated system, which is called upon to supply power to the electrical loads in the sector for which it is responsible (distributed power generation).

\section{Results}

In this section, the results obtained by the simulation of three different cases are reported.

\subsection{Case 1}

The hotel load of the entire ship is served by a single, large plant, which is applied in the first scenario, where the volume and cost of the systems have a higher priority than the emissions.

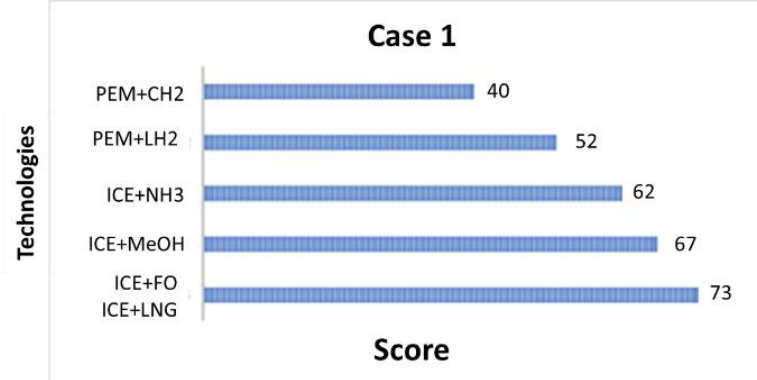

Fig. 5 HELM resulting scores (case 1)

Observing the scores, solutions with $\mathrm{H}_{2}$ and fuel cells are detached from endothermic engine solutions. The "ICE+FO" technology stands out due to its low weight, cost and volume. The "ICE $+\mathrm{LNG}$ " technology manages to compensate the lower score in volume and weight with better environmental performance, thus achieving the same score as the fuel oil engine.

The "ICE+MeOH" and "ICE+NH $\mathrm{N}_{3}$ " innovative technologies are ranked second and third respectively. As mentioned, methanol and ammonia have low energy densities: for the same stored energy, the use of these fuels requires a higher storage volume than conventional marine fuel oils. For this reason, these two solutions obtain a low score for what concerns the volume. Methanol engines benefit from the low cost, similar to the cheapest solution ('ICE+FO'), while ammonia treatment section has a negative impact on the investment required by the 'ICE+NH3' technology, which receives a lower score in the 'cost' category.

Compared to conventional technology, the use of a methanol engine provides a modest reduction in carbon dioxide emissions, while NOx is significantly lower. The ammonia engine is disadvantaged by a high level of NOx emissions (equal to conventional marine fuel oils) but largely offsets this with zero $\mathrm{CO}_{2}$ emissions. 
The lowest scores are obtained by fuel cell technologies "PEM+LH2" and "PEM+CH2". The volume, weight and cost are greatly disadvantaged by the storage units used to store high-density hydrogen (by liquefaction at $253{ }^{\circ} \mathrm{C}$ in the first case, by pressurization up to 700 bar in the second). Fuel cells guarantee the zero-emission of pollutants, but this aspect is little valued in the scenario considered, in which the most compact and convenient solutions such as those with endothermic engines are preferred.

\subsection{Case 2}

In this case, a single generation system is still considered, but the plant is called to operate in a context where pollutant emissions are strictly controlled.

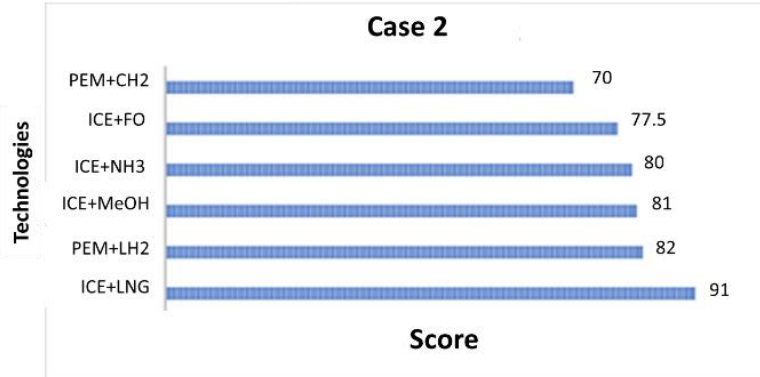

Fig. 6 HELM resulting scores (case 2)

The "ICE+LNG" technology, already encouraged in the previous case by the relatively small size and weight and the cost in line with the most convenient solution, is now further enhanced by the reduced $\mathrm{CO}_{2}$ emissions (the best after fuel cells and ammonia engine) and the lowest amount of NOx produced among endothermic engine solutions. Fuel cells with $\mathrm{LH} 2$ are able to compensate the lower performance offered in terms of volume, cost and weight with the total absence of pollutant emissions, enhanced by the greater relevance given to the environmental parameter in the assumed scenario. The "PEM+LH2" technology is, therefore, able to compete with methanol, ammonia and fuel oil engines, which are generally lighter, cheaper and more compact, but disadvantaged by a regulatory context that strictly controls emissions. The "PEM+CH2" technology also benefits from the scenario considered, but the points earned for environmental performance are not sufficient to compensate for the particularly low scores obtained in the "volume" and "weight" categories.

\subsection{Case 3}

Compared to the previous case, the scenario remains unchanged, but the required power is split into several smaller generation units, which supply power to the users of the MVZ where they are installed.

The energy balance carried out on the cruise ship showed that the total power required is equally distributed in the seven MVZs. The installation of 7 separate systems is assumed, each with a power of 1290 $\mathrm{kW}$. In this case, the ship size selector is set to "Medium", to take into account the lower space provided by the sector in which the system has to be installed.

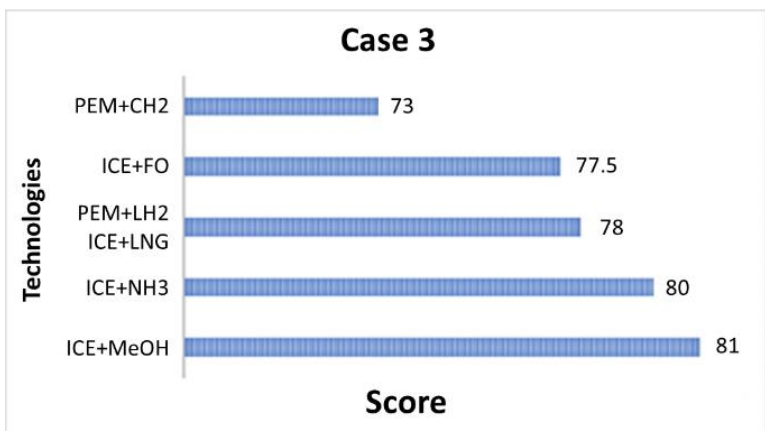

Fig. 7 HELM resulting scores (case 3)

Methanol and ammonia engines take advantage of the fractionation of the plant. In particular, the methanol ICE receives the highest scores for weight and cost, both in line with the values reported by the lightest and most convenient technical solution.

Methanol is a liquid fuel, thus it can be stored in nonpressurized tanks, which can be adapted without any particular problems to the size reduction of the plant. For this reason, the score obtained in the "volume" category does not change compared to previous cases.

The units required for the storage and dissociation of ammonia are heavier, bulkier and more expensive than the "ICE+MeOH" solution, but the better environmental performance allows the "ICE+NH3" solution to obtain a total score slightly lower than the methanol engine, proposed by the software as the recommended solution. "PEM+LH2" technology is penalised by its weight and total volume. Fuel cells are heavier and bulkier than the endothermic engine; the picture is completed by the higher weights and overall dimensions of the hydrogen storage units. The LNG engine is heavily penalized by the fractionation of the plant, which highlighted the weight, the volume occupied and cost of the storage units, more suitable for large applications. As far as the fuel oil solution is concerned, the regulatory context considered favours this engine, which is disadvantaged by the highest pollutant emissions recorded: for this reason, the engine powered by fuel oil occupies the fourth place. In any case, it should be noted that the score achieved by this technology is close to the best solutions, which are therefore able to establish themselves in the scenario considered with a modest margin of competitiveness.

It is appropriate to highlight how the results obtained from the simulation show that distributed power generation involves greater volumes as well as greater investment, due to aspects such as the use of more material, higher workforce costs, etc. However, the advantages of fractioning the electricity generation system should not be underestimated: the management flexibility increases, leading to lower consumptions; furthermore there is an enhancement of the safety on board: the distributed configuration allows for limiting the inconvenience caused by the possible failure of a single section [7].

\section{Conclusions}

In this study, the HELM tool, developed by the authors' research group, is employed in order to perform a 
preliminary design of energy systems onboard, considering cruise ships application in three different scenarios. HELM allows for comparing different technologies related to power generation for propulsion and related fuel storage systems onboard. The different technologies are compared considering four aspects: (i) volume; (ii) weight (iii) cost (iv) emissions. This study focuses on ammonia and methanol used in ICEs, as they represent an interesting compromise between the abovementioned four aspects, having lower emissions than marine fuel oils and higher energy density compared to gaseous fuels. For each case, the considered plant must satisfy the hotel load of the cruise ship (9 MW) for a longstop in port (assumed equal to 30 hours). The results allow the following conclusions to be drawn:

- Considering the actual scenario, with limited importance to environmental impact (case 1), the best solutions are ICE fuelled by FO or LNG, which are already employed solutions. However, the use of $\mathrm{MeOH}$ and $\mathrm{NH} 3$ obtains good scoring, close to the best solutions;

- Considering a close-to-midterm future scenario, with a large relevance to pollutants and $\mathrm{CO}_{2}$ emissions (case 2), the solution with $\mathrm{ICE}+\mathrm{LNG}$ seems the most favourable one, but the solutions with ammonia and methanol obtain high scores as well, becoming more competitive than the ICE+FO solution, which is the most employed nowadays;

- Considering a distributed generation approach and assuming a small size power generation and storage system in each zone of the cruise ship (Case 3), the results change. In this configuration, solutions with ammonia and methanol obtain the highest scores, with a slight advantage on ICE + LNG and fuel cells with LH2.

In conclusion, both the proposed solutions have proven to be competitive in all the investigated scenarios, thus they represent a good compromise between space occupation, costs and environmental aspects.

\section{References}

[1] https://stats.unctad.org/handbook/MaritimeTransp ort/WorldSeaborneTrade.html, access 20/5/2020.

[2] DNV-GL Maritime, Maritime forecast to 2050 Energy transition outlook (2019).

[3] http://www.imo.org/en/MediaCentre/HotTopics/G HG/Pages/default.aspx, access 20/5/2020.

[4] Nian V., Yuan J., A method for analysis of maritime transportation systems in the life cycle approach - The oil tanker example, Applied Energy, 206 (2017), 1579-1589.

[5] American Bureau of Shipping (ABS), setting the course to low carbon shipping (2019).

[6] Blanco H., Nijs W., Ruf J., Faaij A., Potential for hydrogen and Power-to-Liquid in a low-carbon EU energy system using cost optimization, Applied Energy, 232 (2018), 617-639.
[7] Rivarolo M., Rattazzi D., Magistri L., Best operative strategy for energy management of a cruise ship employing different distributed generation technologies", Int. J. of Hydrogen Energy, 43 (2018), 23500-23510.

[8] Rivarolo M., Rattazzi D., Lamberti T., Magistri L., Clean energy production by PEM fuel cells on tourist ships: a time-dependent analysis, Int. J. of Hydrogen Energy, 2020.

[9] www.tpg.unige.it, access 20/5/2020.

[10] Ferrari M.L., Cuneo A., Pascenti M., Traverso A., Real-time state of charge estimation in thermal storage vessels applied to a smart polygeneration grid, Applied Energy, 206 (2017), 90-100.

[11] http://www.methanol.org/wpcontent/uploads/2016/07/Updates-from-StenaGermanica-Per-Stefenson.pdf, access 20/5/2020.

[12] Forno A., Sustainable technologies and fuels for clean energy production in maritime applications, Master Thesis, University of Genoa, 2020.

[13] https://www.fincantieri.com/en/products-andservices/cruise-ships/costa-diadema/ access 20/5/2020. 